\title{
The Physiology of the Tunnel
}

\author{
Susan J. Blackmore \\ Tom S. Troscianko \\ University of Bristol
}

\begin{abstract}
Several theories to account for the origin of tunnel hallucinations and tunnel experiences near death are considered: (1) the idea of a "real" tunnel; (2) representations of transition; (3) reliving birth memories; (4) imagination; and (5) physiological origins. Three different physiological theories are considered that relate the tunnel form to the structure of the visual cortex. All can account for much of the phenomenology of the tunnel experience, and all lead to testable predictions. It is argued that the tunnel experience involves a change in the mental model of the self in the world. Because of this, an experience of purely physiological origin, with no implications for other worlds or for survival, can nevertheless produce lasting changes in the sense of self and reduce the fear of death.
\end{abstract}

The tunnel is dark. The light at the end is bright, beautiful, and alluring. We want to reach the light. It is coming nearer.

The tunnel experience is exciting and somehow mysterious. It gives the impression of mysteries about to be solved or secrets about to be revealed. And yet it seems likely that its origin lies in the structure of the visual cortex. We suggest that these two aspects are not incompatible. We shall explain possible physiological origins of the tunnel and explore the reasons for its numinous qualities.

Here is an example of a tunnel experience occurring as part of a neardeath experience (NDE). It was reported to us by a 70-year-old widow and occurred in 1960 when she was operated on for a spinal tumor.

Dr. Blackmore and Dr. Troscianko are at the Perceptual Systems Research Centre Department of Psychology, University of Bristol. Requests for reprints should be addressed to Dr. Blackmore at the Department of Psychology, University of Bristol, 8-10 Berkeley Square, Bristol BS8 1HH, England. 
Suddenly amidst all this pain (I was still in the dark) I saw a light very faint and in the distance. It got nearer to me, and everything was so quiet; it was warm, I was warm, and all the pain began to go. When I finally stood out of the dark, and into this light, it was the most beautiful thing I have ever seen: soft, warm, translucent. I was finally there, and I felt as if someone had put their arms around me. I was safe, no more pain, nothing, just this lovely, caring sensation.

Next thing I remembered was a doctor or somebody twisting my cheek and several people doing things to me.

This happened again (I think once). Then my husband was again visiting me with my children. I was only vaguely aware of them. I drifted off. I could again see this light. I couldn't wait to go back down that tunnel.

\section{Tunnel Hallucinations}

As early as 1905 Ernest Dunbar collected cases of tunnel experienced under anesthetics and with other drugs (Dunbar, 1905). The tunnel is also one of the form constants noted by Heinrich Kluver in the 1930s (Kluver, 1967). He claimed that almost all hallucinations, regardless of their cause, took on similar basic forms. The four he listed were (1) grating, lattice, fretwork, filigree, honeycomb, or chessboard design; (2) tunnel, funnel, alley, cone, or vessel; (3) spiral; and (4) cobweb. He also noted that these simple hallucinations were extremely bright and the colors highly saturated.

These hallucinations can occur in widely different conditions from hypnagogic imagery when falling asleep (Schachter, 1976); the auras of epilepsy, migraine, or insulin hypoglycemia (Cowan, 1982); and with hallucinogenic drugs, such as LSD or mescaline (Siegel, 1977). Needless to say, the tunnel also occurs as an important part of the NDE (Moody, 1975; Ring, 1980). Interestingly, the other forms do not. The NDE seems to include tunnels, funnels, alleys, cones, and possibly vessels. It also includes great voids of blackness and space, but no gratings and cobwebs. The spiral, of course, may form a kind of tunnel, but we have not come across any examples of a dying person passing through a cobweb or dancing on a chessboard.

This provides an interesting question. Why is it the tunnel alone that seems to be so important in NDEs? Should we ignore this difference and seek one explanation for all the kinds of hallucination; should we assume that all the forms arise from similar causes and then seek to explain why NDEs do not include gratings and cobwebs; or should we seek entirely separate explanations for NDEs and other hallucinations, such that NDEs provide a glimpse into another world? We shall return to this question having considered some of the available evidence. 
Kevin Drab (1981) studied 71 tunnel experiences obtained from 1,112 reports of unusual experiences. He excluded descriptions of voids and black spaces and defined the tunnel as "the perception of a realistic enclosed area of space much longer than its diameter" and found examples occurring in cardiopulmonary arrest; severe stress (from disease or trauma); mild stress (including minor injuries and pain, fatigue, fear, mild fever or toxic conditions, and migraines); and normal conditions (including relaxation, sleep, meditation and hypnosis). Many of the latter category were associated with out-of-body experiences (OBEs).

He found, among other things, that the tunnel was more frequent in serious medical conditions than in nonserious conditions. Also, as one might expect, many of the serious cases involved heart attacks or mechanical accidents. However, there were no cases with cancer or stroke. Since those two conditions rank second and third among the leading causes of death in Americans, this is surprising. It is also known that patients with cancer do report mood elevations and visions of other worlds (Osis \& Haraldsson, 1977). Drab concluded that tunnel experiences are more often triggered by a sudden change in physiological state such as an abrupt drop in blood pressure or shock.

The tunnels were usually dark or dimly lit (only $10 \%$ were brightly lit), and none of the experiencers reported touching the sides, although these varied widely and included scintillating darkness, luminous vapor with fine lines, and bricks with a cobblestone floor. Before my (SB) own first OBE I went down a tunnel made of leaves (Blackmore, 1982a) and I have often experienced tunnels made of varying textures, bright lights, or bands of darker and lighter gray.

Nearly half of Drab's cases reported a light at the end of the tunnel. And $73 \%$ of those described it as becoming larger in their field of vision and described themselves as moving towards it. Many described the light as extremely bright and some even commented that it did not seem to hurt their eyes. Almost all of Drab's experiencers described moving through their tunnels; most went through, some up, and some down, but only eight cases described coming back through the tunnel. It is not clear from Drab's description whether they came backwards through the tunnel or turned around and moved forwards in the opposite direction.

\section{Explaining the Tunnel Experience}

What kind of explanation are we looking for? We think we need to answer this before considering any offered explanations. Our own priorities are that first the theory should account well for the phenome- 
nology. This means explaining why there is a tunnel and not something else, why it is like it is, why there is a light at the end, and so on; and above all, why it seems so real. Second, the theory should not multiply other worlds or bodies or vibrations ad hoc. Third, it should provide testable predictions and the means for changing and improving the theory by experiment. Applying these criteria means that untestable occult theories that can "explain" everything but can never be refuted are not helpful. No more helpful are dismissive theories of the kind "It's all imagination" or "It's all in the mind." Of course, it may all be in the mind, if that phrase means anything, but this is certainly no explanation. So a successful theory must account for the phenomena in ways that are economical and testable.

There are generally four types of explanation offered for the tunnel experience.

\section{A "Real" Tunnel}

In some occult systems there is said to be an actual tunnel that leads from one world to the next.

The problems with such theories are many. If the other worlds are somehow part of this world, then we should be able to measure them or in some way detect their presence. Attempts to do this have notably failed (Blackmore, 1982a). On the other hand, most exponents argue that the higher worlds are in some other dimension or different plane. If these planes and dimensions are not commensurate with the physical world then all the problems of any brain-mind dualism are raised. How can anything be said to pass from one world to another? Positing a tunnel between them certainly does not help. Such theories can neither explain the phenomena, except in a purely ad hoc way, nor provide any predictions by which they might be tested or improved.

\section{Representation of Transition}

An alternative is to say that the tunnel is symbolic of the transition from one state of consciousness to another. Robert Crookall (1964) wrote that there are at least three "deaths" as first the physical, then the soul, and finally the spiritual body are shed to unveil the Eternal Self. The tunnel is a blacking out of consciousness as it passes from one state to another. Celia Green (1968) proposed the tunnel as a representation of a long journey and Kenneth Ring (1980) considered the tunnel 
a psychological phenomenon through which the mind shifts from its normal state of consciousness to a holographic or four-dimensional consciousness of pure frequencies.

This idea escapes the obvious problems of positing "real" tunnels and allows for more flexibility. But in the process we suggest that it loses all power as an explanation. It simply begs the question "Why the tunnel?" Why shouldn't something else be used as a symbol of transition? There could be gates, doorways, arches, chasms, or the great river Styx. In fact these other forms do occur later on in NDEs, in the stage of worlds beyond, but it is the tunnel that appears regularly, in predictable forms, and, as we have seen, in numerous different circumstances. We understand this ubiquitous tunnel no better by saying that it is symbolic of something else.

\section{Reliving Birth Memories}

One of the most popular theories is that the NDE is a reliving of one's birth and that the tunnel is really the birth canal (Honegger, 1983; Sagan, 1977). This theory has been roundly criticized already (Becker, 1982). The main problems are that it is implausible to suppose that the infant would perceive the world in a form that could later be recalled by an adult with totally different perceptual capabilities. Also the birth canal is nothing like a tunnel with a light at the end, and in any case the fetus is pushed along it with the top of its head usually emerging first, not its eyes. It takes a vast leap of the imagination to make the two comparable, and yet this theory has produced a welter of New Age ideas and techniques.

One virtue of this kind of theory is that, at least in some forms, it is testable. If the tunnel experience is reliving birth memories, then the kind of birth should make a difference. In particular, people born by Cesarean section have never been along the birth canal and so, presumably, should not be able to relive it. One of us carried out a survey of 254 people, of whom 36 had been born by cesarean section. These 36 did not report more or fewer tunnel experiences than the others; $36 \%$ of each group reported tunnel experiences (Blackmore, 1982b).

To circumvent this difficulty, Scott Rogo (1982) has argued that the tunnel is not actually reliving one's birth but is a kind of symbolic representation of birth in general. Indeed Carl Sagan's theory can be interpreted in this way. If so, this theory is equivalent to the previous kind of theory and suffers from the same weakness.

Rogo did, however, suggest that people who had a difficult birth 
would have more negative associations with later OBEs than people who had an easy birth. As far as we know this has not yet been tested.

\section{Imagination}

A lot of commentators seem to like the idea that the tunnel, the OBE, and many other experiences are "just imagination." We refer to this approach as a dismissive nonexplanation (Blackmore, 1988). The experiences almost certainly are, or include, the imagination, but this alone is no explanation. If specific similarities between imagination and the tunnel experience can be found, then this is helpful, but we must remember the criteria for a useful theory. The "just imagination" theory neither accounts for the phenomenology (why the dark tunnel with a light at the end, and not a green gate with a ditch in front of it, and so on), nor is it really testable. It can, we suppose, be refuted by the demonstration of something else being involved, but it cannot be improved on by progressive tests or positive predictions. It is, by its very nature, a contentless explanation. For that reason we want a theory that, even if it says the tunnel is imaginary, explains why one should imagine tunnels rather than anything else.

\section{Physiological Explanations}

If we suppose that the hallucinatory form constants are generated by some physiological process, where should we look for it? There is good reason to think they are generated in the cortex. First, according to Cowan (1982) they do not move with the eyes and are therefore not afterimages or other retinal effects. Second, they can be produced by pressure on the eyes. In this case they do move with the eyes but can only be produced by binocular pressure, which again points to the cortex as the site of origin. There are now a number of theories that relate the tunnel form to the structure of the visual cortex.

John Cowan (1982) argued that because we know the appearance of the hallucinations and also the way images on the retina are mapped onto the cortex (Daniel \& Whitteridge, 1961), we should be able to calculate the cortical form that corresponds to any hallucination. Using this mapping he showed that concentric rings on the retina or in the visual world correspond to straight lines parallel to one axis in the visual cortex. Straight lines at right angles to those are mapped in the visual cortex as radiating lines; straight lines at other angles are 
mapped as spirals. If the lines move, the spirals or rings expand and contract. Expanding concentric rings could produce the impression of moving through a tunnel.

But why should there be moving stripes across the visual cortex? Cowan offered an analogy with thermodynamics. In its normal stable state, cortical activity is inhibited by many inhibitory neurons. When these neurons are disrupted, as they are known to be by drugs such as LSD and by anoxia, the disinhibition destabilizes the uniform state, and stripes of activity pass across the cortex. The analogy is with a fluid heated from below, in which either hexagonal patterns or stripes of rising and falling fluid are produced. Cowan concluded that a similar process takes place in the cortex and gives rise to the four form constants.

This theory seems to have some problems. First, it does not account for the fact that NDEs include tunnels but not cobwebs and lattices. It does not explain why people seem to move forwards through tunnels but rarely backwards. Nor does it explain just what those stripes are and why and how they move as they do.

One of us (SB) therefore suggested a far simpler theory. Perhaps no stripes are needed at all. When the brain is starved of oxygen, inhibition is first suppressed, which creates a state of hyperactivity. The cells in the visual cortex will be firing randomly, or noisily. Using the same retinocortical mapping, we can see that there will be far more cells firing that represent the center of the visual field and far fewer at the edges. The effect will appear like a flickering speckled world that gets brighter and brighter towards the center. It is known that the visual system is biased towards movements in an outward direction (Georgeson and Harris, 1978). In addition, visually perceived movement, especially in the absence of any reference, is easily interpreted as self movement; the classic example of this is the feeling that your train is moving backwards when another train pulls out of the station. In other words, this scintillating speckled world of electrical noise could appear to expand outwards from a brighter center. Could this be the tunnel?

Tom Troscianko then made a further suggestion (Blackmore \& Troscianko, 1988). Perhaps this process would not be enough to produce the tunnel effect. However, if you started with very little noise and it gradually increased, the effect would be of a light at the center getting bigger and bigger and hence closer and closer. The tunnel would occur as the noise levels increased and would stop either when they decreased again or when the whole cortex was so noisy that the light enveloped it all. In other words, one would have entered the light. It could get no brighter. 
All these theories have some advantages in common. They explain why there is a tunnel rather than any other symbol of passage to another world. They explain how the light can be extremely bright but does not hurt the eyes: the eyes are not involved at all. The stimulation in the cortex may be as bright, or even brighter, than any real stimulation could actually produce. They all explain Drab's findings that the tunnel occurs with more serious medical conditions, and that it does not occur with slow progressive disease.

\section{Predictions from the Theories}

All the physiological theories provide testable predictions. For example, they imply that an intact visual cortex is required, and if this were damaged, as in some kinds of blindness or stroke, then the tunnel could not be produced.

In other ways they differ. Primarily, Cowan's theory requires that there be stripes of activity passing across the cortex. This might be related to cortical spreading depression, which is thought to be implicated in migraine. This spreads at a rate of 2 to $3 \mathrm{~mm}$ per min (Leao, 1944). By contrast, Cowan claimed that tunnel hallucinations fill up the visual field in about $2.5 \mathrm{msec}$, corresponding to a propagation velocity of 1 or $2 \mathrm{~cm}$ per sec. However, he gave no data or reference for his claim, and that is clearly something to be investigated further. Whether or not the mechanism is similar to cortical spreading depression, it would be expected to have a rate dependent on the neural mechanisms underlying it. In this case, we should expect, according to Cowan's theory, that the tunnel would always be travelled at roughly the same speed. This appears not to be the case. Some people claim to float gently down tunnels, while others rush at a tremendous rate. Drab (1981) reported 67 cases describing movement, of which 12 said they were moving at a slow to moderate speed and 24 were moving very fast.

By comparison, Blackmore's theory makes no stipulation about speed. If the movement is induced merely by the speckly noise, then it depends on the amount of noise: the more noise, the greater the speed. But this in turn implies that faster movement is associated with a larger central white area. As far as we know, this has not been tested.

The final theory makes a clear prediction. If the movement is created by the expansion of the central white area, then speed is not restricted, but the overall change in the tunnel is. In other words, you can only move from a tiny white light to a completely enveloping one. The faster 
you move through the tunnel, the quicker the experience will end. This also has not been tested. So these differences provide a way of testing the various theories, which could be done by collecting appropriate descriptions from people who have experienced the tunnel.

There are other predictions made by these theories. If the key to the tunnel is cortical disinhibition, then the drugs that produce tunnels should all be those that reduce inhibition. This certainly appears to be the case, the major hallucinogens being the best example. On the other hand, some drugs increase inhibition, such as the minor tranquilizers like Valium, which act by potentiating the neurotransmitter gammaaminobutyric acid. We should therefore not expect to find tunnel experiences in people taking these drugs. In particular, if they approach death by an overdose of such drugs they should not have the traditional near-death tunnel. Again, this prediction has not been tested.

We would like now to return to the question of why tunnels occur when they do. With the major hallucinogenic drugs tunnels are common, but so are lattices, spirals, cobwebs, and other simple forms. The tunnel seems to have a special place in the NDE. Why is this?

As far as we can tell, Cowan's theory provides no answer. If the disinhibition is the same regardless of its immediate cause, then the patterns produced should be the same. On the other hand, both the other theories can only produce tunnel forms, and not the other form constants. In particular, Troscianko's theory suggests that the tunnel can only occur when there is a fairly rapid increase in cortical noise, as would be expected in cardiopulmonary failure or an accident or sudden severe stress.

In addition, all these theories can account for Drab's apparently odd finding that there were no tunnels reported by stroke victims. They all require an intact visual cortex, and if that were damaged by a stroke the tunnel could not be seen. A further prediction is related to this. If the tunnel is of cortical origin, then anyone with a damaged visual cortex should not experience it. So, for example, people blind through retinal disease or other damage in the eye should have tunnel experiences just like anyone else, but those suffering from cortical blindness should not. This too awaits testing.

The first way to test these theories should be to find out just what kind of visual stimulation is necessary to induce the impression of moving through a tunnel. Obviously, we cannot open up someone's cortex and apply the hypothesized stimulation that way. We can, however, mimic that stimulation by presenting visual forms to people. Using retinocortical mapping we can construct the visual equivalent of the different kinds of cortical stimulation. In Cowan's case this would 
be concentric rings or spirals. In Blackmore's case it would be randomly flickering specks of light distributed like the cells in the visual system, with many in the center and fewer towards the periphery. In Troscianko's case it would mean having brightness at any spot scaled according to the same mapping, with the brightest in the center fading out to the edges, and the brightness of the whole picture gradually increasing.

So far we have only tried the latter. Troscianko and his colleagues wrote a program to display this on a monitor and it does indeed appear like a tunnel with a bright light at the end that gets gradually brighter (and apparently nearer) (Blackmore \& Troscianko, 1988). This research is still underway, and the next stage is to produce the other forms and to get people who have had tunnel experiences to compare them with their experienced tunnels. This might give some further clue as to the precise origin of the tunnel.

It can be seen that these physiological approaches to the tunnel experience already account for many of the previous findings, and they provide numerous ways of testing them for the future. In this respect they are quite different from all the previous theories I have considered.

\section{Why Is the Tunnel So Real?}

This is a question most physiological theories leave untouched. Nevertheless, the experience cannot be fully understood without considering it.

When tunnels appear in drug-induced states, they are usually considered to be hallucinatory or illusory (Siegel, 1977); but near death, and in some other OBEs, they seem to be as real as anything in normal perception. Why? To answer this we have to step back to the question of why anything ever seems real. It seems implausible to suppose that the perceptual system can easily discriminate input from recalled information when the two are mixed almost from the very periphery. Therefore the system must, at some level, make a decision about which of its representations, or mental models, are "real" and which imaginary. We suggest that it does this at a high level, comparing representations of the world and choosing the most stable as the outside world; i.e. attributing reality to it (Blackmore, 1984, 1988).

Normally, of course, the model chosen is that which is constructed on the basis of input. This is the only one that is stable and predicts future 
input as it goes along. Other models, by comparison, are fleeting and unstable. However, the conditions that give rise to tunnel experiences, as well as OBEs, are precisely those in which input is disrupted, either because of damage to the nervous system or because of deep relaxation, meditation, or sensory isolation. In these conditions the input model is no longer the most stable, and therefore, according to my hypothesis, whichever model is most stable will take over as "reality."

If there is noise in the visual cortex producing a tunnel form, and if the input-driven model is also unstable, then the tunnel form will be the most stable model in the system and hence will be chosen as "real." This is why tunnels near death, but not in the milder drug-induced experiences, seem real. Indeed they are "real" in just the same sense as anything ever is real; because they are the most stable model the system has at the time.

We would take one further step from this, although it is not necessary to understanding the tunnel experience. That is to say that these mental models are not something we construct. Rather "we" are the mental models constructed by the brain. One of us has argued elsewhere (Blackmore, 1989) that consciousness is simply what it is like being a mental model, and the sense of a separate self arises from the construction of a model of a separate self. In other words, the whole system produces a mass of models, and we are just one of them. The normal state of consciousness is dominated by a stable model of self in the world. In the tunnel experience, the tunnel replaces the model of the outside world. It does not necessarily obliterate the model of self.

However, when the tunnel occurs as part of the NDE, it may also involve the dissolution of the self model.

\section{Why the Sense of Mystery?}

More than 80 years ago Dunbar gave a marvelous account of the effects of anesthetics, which were then primarily ether and chloroform. He wrote first:

I have made a point of asking patients in the surgical wards how they felt when they were being anaesthetised. The common experience (eighty per cent of cases) is that of rushing into a dark tunnel. There is singing in the ears and a flashing of lights in the eyes. (Dunbar, 1905, p. 75) 
He also described experiments with ether:

Next comes the sensation that the body is just as much a part of the environment as anything else, and it is perhaps this sensation which, together with the wide-awake intelligence, compels the individual to adopt the standpoint of subjective idealism; which, in its turn, drives him to think that at last the solution of the mystery is dawning upon him. (p. 73)

Isn't this how it is in some drug experiences, in OBEs, and near death? In these states it seems as though the mystery is less impenetrable than before.

This may not be mere delusion. Rather, given what we have said about mental models and consciousness, we think something quite important has happened. Our usual assumption, that the input-based model is a true picture of a "real" world "out there" and that we are a separate individual inhabiting that world and our bodies, is challenged. It becomes obvious that there are other ways of being aware. Any model can seem real. Our assumptions about the real world are shaken. It is this, we suggest, that gives rise both to the impression that "the light is dawning" and to the aftereffects of the experiences on people's lives.

It is misleading that this metaphorical light is often equated with the physiologically induced light. The light at the end of the tunnel is induced by randomly firing neurons. It is not just imagination. It has a very definite physiological origin. This is no reason, however, to dismiss the often claimed insights as worthless. The physiologically induced tunnel can be one way of realizing something important about ourselves, a realization that can change our lives: that is, that we are mental models and nothing more. Frightening as this is to our sober everyday selves, it is not the least frightening when directly experienced. The extent to which this insight can be maintained when the experience is over may be the extent to which fear of death is reduced.

Finally, we have hinted that the dissolution of the self model is possible. All this means is that a biological system that is designed to build a model of self ceases to do so. It isn't an easy state to achieve. Strong biological pressures are against it, but it can happen either through long years of training or in extremis. Afterwards the self model appears again. It is hard for "you" (the self model) to remember what it was like not being; so these states are not easily recalled. Nevertheless there can be a lasting effect on the system. The self never seems quite so solid again and the idea of its death is not so frightening. 


\section{Conclusion}

We have presented several theories of the tunnel experience. The various physiological theories seem by far the best able to explain the phenomenology of the experience, to provide testable predictions, and to contribute to our understanding of altered states of consciousness. A great deal of work now needs to be done to determine which, if any, of these is correct.

These theories entail no other worlds and hold out no hope for survival of death. Nevertheless, far from denigrating the tunnel experience, we think they provide a stepping stone to understanding its numinous and life-changing qualities.

\section{Acknowlegements}

Tom Troscianko was funded by the Medical Research Council (UK). We thank Patrick Bougant and Marni Stewart for programming assistance.

\section{References}

Becker, C.B. (1982). The failure of Saganomics: Why birth models cannot explain neardeath phenomena. Anabiosis: The Journal of Near-Death Studies, 2, 102-109.

Blackmore, S.J. (1982a). Beyond the Body. London, England: Heinemann.

Blackmore, S.J. (1982b). Birth and the OBE: An unhelpful analogy. Journal of the American Society for Psychical Research, 77, 229-238.

Blackmore, S.J. (1984). A psychological theory of the out-of-body experience. Journal of Parapsychology, 48, 201-218.

Blackmore, S.J. (1988). Out of the body? In Basil, R. (Ed.), Not Necessarily the New Age: Critical Essays (pp. 165-184). Buffalo, NY: Prometheus Books.

Blackmore, S.J. (1989). Consciousness: Science tackles the self. New Scientist, 122, $38-41$.

Blackmore, S.J., and Troscianko, T.S. (1988). The psychophysics of death. Perception, 17, 419.

Cowan, J.D. (1982). Spontaneous symmetry breaking in large scale nervous activity. International Journal of Quantum Chemistry, 22, 1059-1082.

Crookall, R. (1964). The Techniques of Astral Projection: Denouement after Fifty Years. Northamptonshire, England: Aquarian Press.

Daniel, P.M., \& Whitteridge, D. (1961). The representation of the visual field on the cerebral cortex in monkeys. Journal of Physiology, 159, 203-221.

Drab, K.J. (1981). The tunnel experience: Reality or hallucination? Anabiosis: The Journal of Near-Death Studies, 1, 126-152.

Dunbar, E. (1905). The light thrown on psychological processes by the action of drugs. Proceedings of the Society for Psychical Research, 19, 62-77. 
Georgeson, M.A., \& Harris, M.G. (1978). Apparent foveofugal drift of counterphase gratings. Perception, 7, 527-536.

Green, C.E. (1968). Out-of-the-Body Experiences. London, England: Hamish Hamilton. Honegger, B. (1983). The OBE as a near-birth experience. In Roll, W.G., Beloff, J., and White, R.A. (Eds.), Research in Parapsychology 1982 (pp. 230-231). Metuchen, NJ: Scarecrow Press.

Kluver, H. (1967). Mescal and Mechanisms of Hallucination Chicago, IL: University of Chicago Press.

Leao, A.A.P. (1944). Spreading depression of activity in the cerebral cortex. Journal of Neurophysiology, 7, 359-390.

Moody, R.A. (1975). Life After Life Covington, GA: Mockingbird.

Osis, K., \& Haraldsson, E. (1977). At the Hour of Death. New York, NY: Avon.

Ring, K. (1980). Life at Death. New York, NY: Coward, McCann \& Geoghegan.

Rogo, D.S. (1982). Psychological models of the out-of-body experience. Journal of Parapsychology, 46, 29-45.

Sagan, C. (1977). Broca's Brain. New York, NY: Random House.

Schachter, D.L. (1976). The hypnagogic state: A critical review of the literature. Psychological Bulletin, 83, 452-481.

Siegel, R.K. (1977). Hallucinations. Scientific American, 237, 132-140. 